\title{
ON SPECIAL GENERATORS FOR PROPERLY INFINITE VON NEUMANN ALGEBRAS ${ }^{1}$
}

\author{
W. R. WOGEN
}

\begin{abstract}
It is known that every properly infinite von Neumann algebra $\mathcal{Q}$ on a separable Hilbert space has a single generator. We show in this paper that a generator for $\mathbb{Q}$ may be chosen from some special classes of operators. In particular each of the following classes of operators contains a generator for $a$ : the hyponormals, the nilpotents, the transcendental quasinilpotents, and the unimodular contractions. We also show that a generator for $\mathbb{Q}$ may be chosen with arbitrarily prescribed spectrum.
\end{abstract}

Introduction. Let $\mathfrak{H C}$ be a separable complex Hilbert space. A von Neumann algebra $a$ is properly infinite if $a$ contains no finite projections in its center. If $S$ is an algebra of operators, $S^{\prime}$ denotes the commutant of $\delta$. For $2 \leqq n \leqq \aleph_{0}, M_{n}(\delta)$ denotes the algebra of $n \times n$ matrices over $\mathcal{S}$ which act boundedly on $\sum_{k=1}^{n} \oplus$ Fe. Let $\mathcal{R}(A, B, \cdots)$ denote the von Neumann algebra generated by the set $\{A, B, \cdots\}$ of operators. The reader is referred to [2] and [6] as references on von Neumann algebras.

Throughout this paper we will need the well-known fact that if $a$ is a properly infinite von Neumann algebra, then $a$ is *-isomorphic to $M_{n}(a)$ for $1 \leqq n \leqq \boldsymbol{\aleph}_{0}$ (cf. [6, Corollary 14]). Also, it is known that a *-isomorphism of a von Neumann algebra $a$ onto a von Neumann algebra $B$ carries a generator of $a$ onto a generator of $B$ (cf. [6, p. 68]).

It was shown in [7] that if $Q$ is a properly infinite von Neumann algebra, then $a$ has a single generator. It then follows from [3, Lemma 1] that $a$ has a partially isometric generator. In this note we will construct some other generators for $a$.

The author wishes to thank Professor D. Topping and Professor J. Stampli for many helpful conversations concerning the material presented here.

Results. We begin with a lemma.

Received by the editors May 26, 1970.

AMS 1969 subject classifications. Primary 4665, 4710.

Key words and phrases. Generator, properly infinite von Neumann algebra, hyponormal, transcendental quasinilpotent, unimodular contraction.

1 This paper is part of the author's doctoral dissertation written under the direction of Professor D. Topping. 
LEMMA 1. Let $3 \leqq n \leqq \aleph_{0}$, and let $\left\{a_{k}\right\}_{k=1}^{n}$ and $\left\{\lambda_{k}\right\}_{k=1}^{n}$ be bounded sequences of complex numbers with $a_{k} \neq 0 \quad \forall k$. Let $a$ be $a$ von Neumann algebra with $a=R(A)$, where $A$ satisfies $\left\|\left(\lambda_{1}-\lambda_{2}\right) A\right\|<\left|a_{1} a_{2}\right|$. Define $B=\left(B_{i, j}\right)_{i, j=1}^{n} \in M_{n}(a)$ by $B_{i, i}=\lambda_{i} I, B_{i+1, i}=a_{i} I, B_{3,1}=A$, and $B_{i, j}=0$ otherwise. Then $R(B)=M_{n}(Q)$.

Proof. We will show that $R(B)^{\prime}=\left\{\left(\delta_{i, j} D\right)_{i, j=1}^{n}: D \in Q^{\prime}\right\}$. It then follows easily that $R(B)=R(B)^{\prime \prime}=M_{n}(Q)$. Let $C=\left(C_{i, j}\right)_{i, j=1}^{n} \in R(B)^{\prime}$ with $C$ selfadjoint (i.e., $C_{i, j}=C^{*}{ }_{j, i}$ ). Then $B C=\left(E_{i, j}\right)_{i, j-1}^{n}$, where

$$
E_{1, j}=\lambda_{1} C_{1, j}, \quad E_{3, j}=A C_{1, j}+a_{2} C_{2, j}+\lambda_{3} C_{3, j},
$$

and for $i \neq 1,3$,

$$
E_{i, j}=a_{i-1} C_{i-1, j}+\lambda_{i} C_{i, j}
$$

$C B=\left(F_{i, j}\right)_{i, j=1}^{n}$, where

$$
F_{i, 1}=\lambda_{1} C_{i, 1}+a_{1} C_{i, 2}+C_{i, 3} A,
$$

and $\forall j \neq 1$,

$$
F_{i, j}=\lambda_{j} C_{i, j}+a_{j} C_{i, j+1} .
$$

By assumption, $B C=C B$. Since $E_{1,1}=F_{1,1}$ and $E_{1,2}=F_{1,2}$, we have $a_{1} C_{1,2}+C_{1,3} A=0$ and $a_{2} C_{1,3}=\left(\lambda_{1}-\lambda_{2}\right) C_{1,2}$. It follows that $C_{1,3}\left(a_{1} a_{2} I+\left(\lambda_{1}-\lambda_{2}\right) A\right)=0$. But $\left\|\left(\lambda_{1}-\lambda_{2}\right) A\right\|<\left|a_{1} a_{2}\right|$ so $a_{1} a_{2} I$ $+\left(\lambda_{1}-\lambda_{2}\right) A$ is invertible, and $C_{1,3}=0$. Thus also $C_{1,2}=0$. Now $E_{1,3}=F_{1,3}$ implies $C_{1,4}=0$. Proceeding in this way we get $C_{1, k}=0$ for $k \geqq 2$. Now if we compare $E_{2, j}$ and $F_{2, j}$, we see by similar arguments that $C_{2, k}=0$ for $k \geqq 3$. Continuing in this way we get $C_{i, j}=0 \quad \forall i<j$. $C_{i, j}=C_{j, i}^{*}$, so $C_{i, j}=0 \forall i \neq j$.

Since $E_{i+1, i}=F_{i+1, i}$, we have $C_{1,1}=C_{i, i}$ for $i \geqq 1$. Finally, since $E_{3,1}=F_{3,1}$, we have $A C_{1,1}=C_{3,3} A=C_{1,1} A . C_{1,1}$ is selfadjoint, so $C_{1,1} \in R(A)^{\prime}=a^{\prime}$. It follows that $R(B)^{\prime}=\left\{\left(\delta_{i, j} D\right)_{i, j=1}^{n}: D \in \alpha^{\prime}\right\}$ as asserted.

Definition 1. An operator $A$ is hyponormal if $A^{*} A-A A^{*} \geqq 0$.

Note that hyponormality is invariant under *-isomorphism.

TheOREM 1. If $a$ is a properly infinite von Neumann algebra on a separable Hilbert space, then a has a hyponormal generator.

Proof. Choose $A \in Q$ such that $R(A)=Q$ and $\|A\| \leqq 1 / 2$. Let $B=\left(B_{i, j}\right)_{i, j=1}^{\infty} \in M_{\infty}(a)$ be defined by $B_{2,1}=I, B_{3,2}=2 I, B_{i+1, i}=3 I$ for $i \geqq 3, B_{3,1}=A$, and $B_{i, j}=0$ otherwise. Then by Lemma $1, R(B)$ $=M_{\infty}(a)$. We assert that $B$ is hyponormal. In fact, to show $B^{*} B-B B^{*} \geqq 0$, it suffices to show that the 3 by 3 matrix 


$$
\left[\begin{array}{ccc}
I+A^{*} A & 2 A^{*} & 0 \\
2 A & 3 I & -A^{*} \\
0 & -A & 5 I-A A^{*}
\end{array}\right]
$$

is positive. This is a routine computation. Since $Q$ is ${ }^{*}$-isomorphic to $M_{\infty}(a)$, it follows that $a$ has a hyponormal generator.

This theorem shows in particular that there exist hyponormal operators $A$ such that $R(A)$ is not type I.

We mention that $\mathrm{H}$. Behncke has recently shown [1] that Theorem 1 is true with "hyponormal" replaced by "subnormal".

REMARK 1 . If $A$ is hyponormal and $R(A)$ is finite, then $A$ is normal and $R(A)$ is abelian. (This holds because if $R(A)$ is finite, then there is a unique center valued trace function $\tau$ on $R(A)$ (cf. [2, Chapter III, \$4]) satisfying, in particular, (1) $\tau(C B-B C)=0 \forall B, C \in R(A)$, and (2) if $P \geqq 0$ and $\tau(P)=0$, then $P=0$. Hence if $A^{*} A-A A^{*} \geqq 0$, then $\left(A^{*} A-A A^{*}\right)=0$, so $A^{*} A-A A^{*}=0$, and $A$ is normal.) It follows that if $A$ is hyponormal, then $\Re(A)$ is of the form $\propto \oplus \Theta$, where $Q$ is abelian and $B$ is properly infinite.

Definition 2. An operator $A$ is quasinilpotent if $\lim _{n \rightarrow \infty}\left\|A^{n}\right\| 1 / n=0$. $A$ is nilpotent of index $n$ if $A^{n}=0$ and $A^{n-1} \neq 0 . A$ is transcendental quasinilpotent if $A$ is quasinilpotent but not nilpotent.

REMARK 2. Topping has shown [5, Theorem 3] that a properly infinite von Neumann algebra is linearly spanned by its transcendental quasinilpotents.

THEOREM 2. Let a be a properly infinite von Neumann algebra on a separable Hilbert space. Then a has a transcendental quasinilpotent generator.

ProOF. Let $\left\{a_{n}\right\}_{n=1}^{\infty}$ be a sequence of nonzero complex numbers with $\lim _{n \rightarrow \infty} a_{n}=0$. Let $A$ be a generator of $Q$. Define $Q=\left(Q_{i, j}\right)_{i, j=1}^{\infty} \in M_{\infty}(Q)$ by $Q_{i+1, i}=a_{i} I, Q_{i, j}=0$ otherwise. Then $Q$ is a weighted shift, and it is easy to show that $Q$ is quasinilpotent. Let $N=\left(N_{i, j}\right)_{i, j=1}^{\infty} \in M_{\infty}(Q)$ be defined by $N_{3,1}=A, N_{i, j}=0$ otherwise. Let $B=Q+N$. Then $R(B)$ $=M_{\infty}(\mathrm{Q})$ by Lemma 1 . We claim that $B$ is a transcendental quasinilpotent. In fact, a computation shows that $B^{n}=(Q+N)^{n}=Q^{n}$ $+Q^{n-1} N$. Thus $\left\|B^{n}\right\|^{1 / n}=\left\|Q^{n}+Q^{n-1} N\right\|^{1 / n} \leqq\left\|Q^{n-1}\right\| 1 / n\|Q+N\|^{1 / n} \rightarrow 0$ as $n \rightarrow \infty$. Now note that transcendental quasinilpotence is a ${ }^{*}$-isomorphism invariant and that $Q$ is ${ }^{*}$-isomorphic to $M_{\infty}(Q)$. It follows that $a$ has a transcendental quasinilpotent generator.

THEOREM 3. If $n \geqq 3$ and $a$ is a separably acting properly infinite von Neumann algebra, then a has a nilpotent generator of index $n$. 
Proof. If $a=R(A)$, let $B=\left(B_{i, j}\right)_{i, j=1}^{n} \in M_{n}(a)$ be defined by $B_{i+1, i}=I, B_{3,1}=A$, and $B_{i, j}=0$ otherwise. Then $R(B)=M_{n}(Q)$ by Lemma 1 , and $B$ is nilpotent of index $n$. $Q$ is ${ }^{*}$-isomorphic to $M_{n}(a)$, so the theorem follows. (For $n=3$, this theorem is due to Pearcy and Ringrose (unpublished).)

Let $\sigma(A)$ denote the spectrum of $A$. The next theorem asserts that a properly infinite von Neumann algebra has generators with arbitrarily prescribed spectrum. More precisely,

THEOREM 4. Let $a$ be a properly infinite von Neumann algebra on a separable Hilbert space $\mathfrak{H C}$ and let $K$ be a nonempty compact subset of the complex plane. Then there is an operator $B$ in a such that $R(B)=Q$ and $\sigma(B)=K$.

Proof. Let $\left\{a_{k}\right\}_{k=1}^{\infty}$ be a sequence of complex numbers such that $a_{k} \neq 0 \quad \forall k$ and $\lim _{k \rightarrow \infty} a_{k}=0$. Let $\delta$ be a countable dense subset of $K$. Form a sequence $\left\{\lambda_{k}\right\}_{k=1}^{\infty}$ from $S$ such that each element of $\mathcal{S}$ occurs

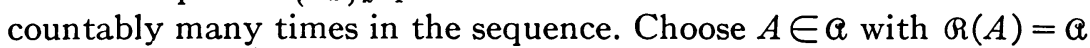
and $\left\|\left(\lambda_{1}-\lambda_{2}\right) A\right\|<\left|a_{1} a_{2}\right|$.

Let $N=\left(\delta_{i, j} \lambda_{i} I\right)_{i, j=1}^{\infty} \in M_{\infty}(\alpha)$. Let $Q=\left(Q_{i, j}\right)_{i, j=1}^{\infty} \in M_{\infty}(Q)$ be defined by $Q_{i+1, i}=a_{i} I, Q_{3,1}=A$, and $Q_{i, j}=0$ otherwise. Write $B=Q+N$. Then $B$ is an operator on $\sum_{k=1}^{\infty} \oplus \mathfrak{H}_{k}$, where $\mathfrak{H}_{k}=\mathfrak{H C} \forall k$. By Lemma 1 , $\mathcal{R}(B)=M_{\infty}(Q)$. We will show that $\sigma(B)=K$.

Notice that $\lambda \in \sigma(B) \Leftrightarrow 0 \in \sigma(B-\lambda)$, and that the matrix $B-\lambda$ has the same form as $B$. Hence it suffices to show that $0 \in \sigma(B)$ $\Leftrightarrow 0 \in K=\left\{\lambda_{k}\right\}$ -

Suppose first that $0 \in\left\{\lambda_{k}\right\}-$. Then there is a subsequence $\left\{\lambda_{k_{j}}\right\}_{j=1}^{\infty}$ such that $\lim _{j \rightarrow \infty} \lambda_{k_{j}}=0$. Choose $x_{k_{j}} \in \mathcal{F}_{k_{j}}$ with $\left\|x_{k_{j}}\right\|=1$, and identify $x_{k_{j}}$ with the vector $\left(0,0, \cdots 0, x_{k_{j}}, 0, \cdots\right) \in \sum_{k=1}^{\infty} \oplus \mathcal{F}_{k}$ whose only nonzero entry is in the $k_{j}$ th position. Then $\left\|B x_{k_{j}}\right\| \leqq\left\|\lambda_{k_{j}} x_{k_{j}}\right\|+\left\|a_{k_{j}} x_{k_{j}}\right\|$ $=\left|\lambda_{k_{j}}\right|+\left|a_{k_{j}}\right|$ for $k_{j}>1$. But $\lim _{j \rightarrow \infty} a_{k_{j}}=\lim _{j \rightarrow \infty} \lambda_{k_{j}}=0$, so that $\left\|B x_{k_{j}}\right\| \rightarrow 0$ as $j \rightarrow \infty$. Thus $0 \in \sigma(B)$. (This argument actually shows that 0 is in the approximate point spectrum of $B$.)

Now suppose that $0 \notin\left\{\lambda_{k}\right\}^{-}$. Then $N$ is invertible. In fact, $N^{-1}$ $=\left(\delta_{i, j} \lambda_{i}^{-1} I\right)_{i, j=1}^{\infty}$. Computing $N^{-1} Q$, we find that $N^{-1} Q=\left(C_{i, j}\right)_{i, j=1}^{\infty}$, where $C_{i+1, i}=\lambda_{i+1}^{-1} a_{i} I, C_{3,1}=\lambda_{3}^{-1} A$, and $C_{i, j}=0$ otherwise. Then $N^{-1} Q$ is quasinilpotent by the proof of Theorem 2 , since $\lim _{k \rightarrow \infty} \lambda_{k+1}^{-1} a_{k}=0$. Thus $I+N^{-1} Q$ is invertible. But then $B=N+Q=N\left(I+N^{-1} Q\right)$ is the product of invertible operators, so $B$ is invertible, i.e., $0 \notin \sigma(B)$. The theorem now follows since $Q$ is *-isomorphic to $M_{\infty}(Q)$.

DEFINITION 3. An operator $A$ is a unimodular contraction if $\|A\| \leqq 1$ and $\sigma(A) \subset\{z:|z|=1\}$. 
Note that the image under a ${ }^{*}$-isomorphism of a unimodular contraction is a unimodular contraction. In [4], Russo poses the question: "Do there exist unimodular contractions of type II $_{\infty}$ and III?" The following theorem answers this question in the affirmative.

THEOREM 5. If $a$ is a properly infinite von Neumann algebra on a separable Hilbert space $\mathfrak{H C}$, then a has a unimodular contractive generator.

We first prove a lemma.

Lemma 2. Let $\left\{a_{k}\right\}_{k=1}^{\infty}$ be an increasing sequence of positive numbers such that $\lim _{k \rightarrow \infty} a_{k}=1$. Let the operator $T=\left(b_{i, j}\right)_{i, j=-\infty}^{\infty}$ on $l^{2}(Z)$ be defined by $b_{i, i-1}=1$ for $i \leqq 0, b_{i, i-1}=a_{i}$ for $i>0$, and $b_{i, j}=0$ otherwise. Then $B$ is a unimodular contraction.

Proof. $T$ is a two-sided weighted shift. Obviously $T$ is a contraction. But an easy computation shows that $\left\|T^{-n}\right\|=1 / a_{1} a_{2} \cdots a_{n}$. Then

$$
\lim _{n \rightarrow \infty}\left\|T^{-n}\right\|^{1 / n}=\lim _{n \rightarrow \infty}\left(1 / a_{1} a_{2} \cdots a_{n}\right)^{1 / n}=1,
$$

since $\lim _{n \rightarrow \infty} a_{n}=1$. Thus the spectral radius of $T^{-1}$ is 1 , and it follows that $\sigma(T) \subset\{z:|z|=1\}$.

Proof of Theorem 5. By Theorem 3 , we can choose $A \in Q$ with $\mathfrak{R}(A)=Q$ and $A$ nilpotent. Moreover, we may suppose $\|A\| \leqq 1 / 2$. Let $a_{n}=n /(n+1)$ for $n=1,2, \cdots$. Define $Q=\left(Q_{i, j}\right)_{i, j=-\infty}^{\infty} \in M_{\infty}(Q)$ by $Q_{2,0}=A$ and $Q_{i, j}=0$ otherwise. Define $T=\left(T_{i, j}\right)_{i, j=-\infty}^{\infty} \in M_{\infty}(Q)$ by $T_{i, i-1}=I$ for $i \leqq 0, T_{i, i-1}=a_{i} I$ for $i>0$, and $T_{i, j}=0$ otherwise. Let $B=T+Q$. We claim that $B$ is a unimodular contractive generator for $M_{\infty}(\mathrm{Q})$.

We show first that $B$ is a contraction. Recall that $\|A\| \leqq 1 / 2$, $a_{1}=1 / 2$, and $a_{2}=2 / 3$. Let $x=\left(x_{n}\right)_{n=-\infty}^{\infty} \in \sum_{n=-\infty}^{\infty} \oplus$ ऊ. Then

$$
\begin{aligned}
\|B x\|^{2} & =\sum_{-\infty}^{-1}\left\|x_{n}\right\|^{2}+\left\|a_{1} x_{0}\right\|^{2}+\left\|A x_{0}+a_{2} x_{1}\right\|^{2}+\sum_{2}^{\infty}\left\|a_{n+1} x_{n}\right\|^{2} \\
& \leqq \sum_{-\infty}^{-1}\left\|x_{n}\right\|^{2}+\left\|a_{1} x_{0}\right\|^{2}+2\left(\left\|A x_{0}\right\|^{2}+\left\|a_{2} x_{1}\right\|^{2}\right)+\sum_{2}^{\infty}\left\|x_{n}\right\|^{2} \\
& \leqq \sum_{-\infty}^{-1}\left\|x_{n}\right\|^{2}+\left((1 / 2)^{2}+2(1 / 2)^{2}\right)\left\|x_{0}\right\|^{2}+2(2 / 3)^{2}\left\|x_{1}\right\|^{2}+\sum_{2}^{\infty}\left\|x_{n}\right\|^{2} \\
& \leqq\|x\|^{2} .
\end{aligned}
$$

Thus $B$ is a contraction. 
Next we show that $\sigma(B) \subset\{z:|z| \leqq 1\}$. It suffices to show that if $|\lambda|<1$, then $\lambda \notin \sigma(B)$. Let $|\lambda|<1$. By Lemma 2, $T$ is a unimodular contraction. Thus $T-\lambda I$ is invertible. $B=T+Q$, so $B-\lambda I=(T-\lambda I)$ $+Q=(T-\lambda I)\left[I+(T-\lambda I)^{-1} Q\right]$. Now simple matrix multiplication shows that $(T-\lambda I)^{-1} Q$ is nilpotent. (In fact if $A^{n}=0$ and $C \in M_{\infty}(C I)$, then $(C Q)^{n}=0$.) Hence $I+(T-\lambda I)^{-1} Q$ is invertible. Since $B-\lambda I$ is a product of invertible operators, $B-\lambda I$ is invertible and $\lambda \notin \sigma(B)$.

Finally we sketch a proof that $R(B)=M_{\infty}(Q)$. As in Lemma 1, it suffices to show that $R(B)^{\prime}=\left\{\left(\delta_{i, j} D\right)_{i, j=-\infty}^{\infty}: D \in Q^{\prime}\right\}$. Let $C$ $=\left(C_{i, j}\right)_{i, j=-\infty}^{\infty} \in R(B)^{\prime}$ with $C$ selfadjoint. Then $B C=\left(E_{i, j}\right)_{i, j=-\infty}^{\infty}$, where

and

$$
\begin{aligned}
& E_{2, j}=A C_{0, j}+a_{2} C_{1, j}, \\
& E_{i, j}=C_{i-1, j} \quad \text { for } i \leqq 0,
\end{aligned}
$$

$$
E_{i, j}=a_{i} C_{i-1, j} \quad \text { for } i>0, i \neq 2 .
$$

$C B=\left(F_{i, j}\right)_{i, j=-\infty}^{\infty}$, where

and

$$
\begin{aligned}
& F_{i, 0}=a_{i} C_{i, 1}+C_{i, 2} A, \\
& F_{i, j}=C_{i, j+1} \quad \text { for } j<0,
\end{aligned}
$$

$$
F_{i, j}=a_{j+1} C_{i, j+1} \quad \text { for } j>0 .
$$

We are assuming that $B C=C B$ and that $C_{i, j}=C_{j, i}^{*}$.

Let $n \geqq 3$. Since $E_{0, n-1}=F_{0, n-1}$ and $E_{n,-1}=F_{n,-1}$, we find that $a_{n} C_{0, n}=C_{-1, n-1}$ and $a_{n} C_{-1, n-1}^{*}=C_{0, n}^{*}$. But $a_{n} \neq 1$, so $C_{0, n}=C_{-1, n-1}=0$. Fix $n \geqq 3$. Comparing $E_{k, k+n-1}$ and $F_{k, k+n-1}$, we find that $C_{k, k+n}=0$ $\forall k$. But $E_{2,3}=F_{2,3}$ and $E_{4,1}=F_{4,1}$, so that $a_{2} C_{1,3}=a_{4} C_{2,4}$ and $a_{2} C_{2,4}^{*}$ $=a_{4} C_{1,3}^{*}$. Since $a_{2} \neq a_{4}$, we must have $C_{1,3}=C_{2,4}=0$. It follows that $C_{k, k+2}=0 \quad \forall k$. Similarly, since $E_{2,2}=F_{2,2}$ and $E_{3,1}=F_{3,1}$, we get $a_{2} C_{1,2}=a_{3} C_{2,3}$ and $a_{3} C_{1,2}^{*}=a_{2} C_{2,3}^{*}$. Hence $C_{1,2}=C_{2,3}=0$ and thus $C_{k, k+1}=0 \quad \forall k$. We have shown that $C_{k, n+k}=0 \quad \forall n \geqq 1$ and $\forall k$. But $C_{i, j}=C_{j, i}^{*}$, so $C_{i, j}=0 \quad \forall i \neq j$.

Because $E_{k+1, k}=F_{k+1, k}$, we find that $C_{k, k}=C_{0,0} \forall k$. Finally, since $E_{2,0}=F_{2,0}$, we have $A C_{0,0}=C_{2,2} A=C_{0,0} A$, i.e., $C_{0,0} \in Q^{\prime}$. Therefore $R(B)^{\prime}=\left\{\left(\delta_{i, j} D\right)_{i, j=-\infty}^{\infty}: D \in Q^{\prime}\right\}$. Since $a$ is *-isomorphic to $M_{\infty}(a)$, the proof is complete.

REMark 3. Russo has shown (cf. [4, Theorem 1]) that if $A$ is a unimodular contraction and $R(A)$ is finite, then $A$ is unitary. It follows if $A$ is any unimodular contraction, then $\mathcal{R}(A)$ is of the form $Q \oplus B$, where $Q$ is abelian and $B$ is properly infinite. 


\section{REFERENCES}

1. H. Behncke, Generators of $W^{*}$-algebras (to appear).

2. J. Dixmier, Les algebres d'operateurs dans l'espace Hilbertien (Algebres de von Neumann), Cahiers Scientifiques, fasc. 25, Gauthier-Villars, Paris, 1957. MR 20 \#1234.

3. C. M. Pearcy, On certain von Neumann algebras which are generated by partial isometries, Proc. Amer. Math. Soc. 15 (1964), 393-395. MR 28 \#4380.

4. B. Russo, Unimodular contractions in Hilbert space, Pacific J. Math. 26 (1968), 163-169. MR $37 \# 6769$.

5. D. Topping, Transcendental quasinilpotents in operator algebras, J. Functional Anal. 2 (1968), 342-351. MR 38 \#1535. 1969.

6. - Lectures on von Neumann algebras, Lecture notes, Tulane University,

7. W. Wogen, On generators for von Neumann algebras, Bull. Amer. Math. Soc. 75 (1969), 95-99. MR 38 \#5020.

University of North Carolina, Chapel Hill, North Carolina 27514 